\title{
Article
}

\section{Cesaro Limits for Fractional Dynamics}

\author{
Yuri Kondratiev ${ }^{1,2,+}$ and José da Silva ${ }^{3, *,+} \mathbb{C}$ \\ 1 Department of Mathematics, University of Bielefeld, D-33615 Bielefeld, Germany; \\ kondrat@math.uni-bielefeld.de \\ 2 Institute of Mathematics of the National Academy of Sciences of Ukraine, National Pedagogical Dragomanov \\ University, 33615 Kiev, Ukraine \\ 3 CIMA, University of Madeira, Campus da Penteada, 9020-105 Funchal, Portugal \\ * Correspondence: joses@staff.uma.pt; Tel.: +351-291-705-185 \\ $+\quad$ These authors contributed equally to this work.
}

check for

updates

Citation: Kondratiev, Y.; da Silva, J. Cesaro Limits for Fractional

Dynamics. Fractal Fract. 2021, 5, 133. https://doi.org/10.3390/

fractalfract5040133

Academic Editor: Maja Andrić

Received: 25 August 2021

Accepted: 17 September 2021

Published: 22 September 2021

Publisher's Note: MDPI stays neutral with regard to jurisdictional claims in published maps and institutional affiliations.

Copyright: (c) 2021 by the authors. Licensee MDPI, Basel, Switzerland. This article is an open access article distributed under the terms and conditions of the Creative Commons Attribution (CC BY) license (https:// creativecommons.org/licenses/by/ $4.0 /)$.
Abstract: We study the asymptotic behavior of random time changes of dynamical systems. As random time changes we propose three classes which exhibits different patterns of asymptotic decays. The subordination principle may be applied to study the asymptotic behavior of the random time dynamical systems. It turns out that for the special case of stable subordinators explicit expressions for the subordination are known and its asymptotic behavior are derived. For more general classes of random time changes explicit calculations are essentially more complicated and we reduce our study to the asymptotic behavior of the corresponding Cesaro limit.

Keywords: dynamical systems; random time change; inverse subordinator; asymptotic behavior

\section{Introduction}

In this paper we will deal with Markov processes or dynamical systems in $\mathbb{R}^{d}$. These processes or dynamics starting from $x \in \mathbb{R}^{d}$, denote by $X^{x}(t), t \geq 0$, have associated evolution equations on $\mathbb{R}^{d}$. In the Markov case we define for suitable $f: \mathbb{R}^{d} \longrightarrow \mathbb{R}$ the function $u(t, x)=\mathbb{E}\left[f\left(X^{x}(t)\right)\right]$ which satisfied the Kolmogorov equation

$$
\frac{\partial}{\partial t} u(t, x)=L u(t, x)
$$

where $L$ is the generator of the Markov process.

For a dynamical system we introduce $u(t, x)=f\left(X^{x}(t)\right)$. Then this function is the solution of the Liouville equation

$$
\frac{\partial}{\partial t} u(t, x)=L u(t, x)
$$

where now $L$ is the Liouville operator for the dynamical system, see e.g., Kondratiev and da Silva [1].

Let $S(t), t \geq 0$ be a subordinator and $E(t), t \geq 0$ denotes the inverse subordinator, that is, for each $t \geq 0, E(t):=\inf \{s>0 \mid S(s)>t\}$. This random process we consider as a random time and assume to be independent of $X^{x}(t)$. Define a random process $Y^{x}$ by

$$
Y^{x}(t, \omega):=X^{x}(E(t, \omega)) .
$$

Then as above we may introduce

$$
u^{E}(t, x)=\mathbb{E}\left[f\left(Y^{x}(t)\right)\right]
$$


For both Markov and dynamical system cases this function satisfies the evolution equations

$$
D_{t}^{E} u^{E}(t, x)=L u^{E}(t, x)
$$

where $L$ is the Kolmogorov or Liouville operator correspondingly. Here $D_{t}^{E}$ is a generalized fractional time derivative corresponding to the inverse subordinator $E(t)$, see Section 2 below for details, in particular the definition in (15). The main relation which is true for both cases is the following subordination formula:

$$
u^{E}(t, x)=\int_{0}^{\infty} u(\tau, x) G_{t}(\tau) d \tau,
$$

where $G_{t}(\tau)$ is the density of the inverse subordinator $E(t)$, see, e.g., Toaldo [2], Kondratiev and da Silva [1] and especially the book Meerschaert and Sikorskii [3]. This formula which relates the solutions of the evolution equations with usual and fractional derivatives plays an important role in the study of dynamics with random times. Note that there exist such relations between random times, fractional equations and subordination in the framework of physical models, see, e.g., Mura et al. [4].

The goal of this paper is to study and analyze the asymptotic behavior of two elementary dynamical system after the random time change, namely $u(t, x)=e^{-a t}, a>0$ and $u(t, x)=t^{n}, n \geq 0$. Here the dynamical system are considered as a deterministic Markov processes. For particular classes of random times the subordination formula (1) is evaluated explicitly. This is true, for example, in the case of inverse stable subordinators. For a general inverse subordinator the properties of the density $G_{t}(\tau)$ are unknown and the evaluation of (1) is not possible. Actually, it is a long standing open problem in the theory of stochastic processes.

We propose an alternative approach to study the asymptotic behavior of $u^{E}(t, x)$. More precisely, we consider Cesaro limits (the asymptotic of the Cesaro mean of $u^{E}(t, x)$, see (23) below) of $u^{E}(t, x)$ using the subordination formula representation (1) together with the Feller-Karamata Tauberian theorem, see Theorem 1. For many classes of random times this approach leads to a precise asymptotic behavior. In this paper we investigate three classes of random time change, denote by (17)-(19), see Section 2, which exhibits different patterns of decays of the Cesaro limit of $u^{E}(t, x)$. We would like to emphasize that for particular classes of random times, namely inverse stable subordinators, the asymptotic of $u^{E}(t, x)$ which may be computed explicitly, coincides with the Cesaro limit. For other classes of random times the Cesaro limit gives one possible characteristic of the asymptotic for $u^{E}(t, x)$. To the best of our knowledge at the present time no other information on the asymptotic of $u^{E}(t, x)$ is known for a general subordinator.

The remaining of the paper is organized as follows. In Section 2 we introduce three classes (17)-(19) of subordinator processes which serves as random times. These classes are given in terms of their local behavior of the Laplace exponent at $\lambda=0$. In addition, we state the main results of the paper. Section 3 is a preparation for the more general study of the asymptotic of the subordination in Section 4. More precisely, we investigate in detail the special case of the inverse stable subordinator where explicit expressions are known. Hence, the expression for the subordination (1) is derived (for the two dynamical systems considered above) as well as their Cesaro limit. It turns out that both asymptotic for $u^{E}(t, x)$ (the explicit calculations and Cesaro limit) are the same. Finally in Section 4 we study the Cesaro limit for the general classes (17)-(19) of random time changes.

\section{Random Times Processes}

In this section we introduce three classes of subordinators which serves as random times processes. More precisely, the random times corresponds to the inverse of subordinator processes whose Laplace exponent satisfies certain conditions, see below for details. The simplest example in class (17) below, is the well known $\alpha$-stable subordinators whose inverse processes are well studied in the literature, see for example Bingham [5] or Feller [6]. 
The classes of processes to be introduced which serve as random times have a connection with the concept of general fractional derivatives (see Kochubei [7] for details and applications to fractional differential equations) associated to an admissible kernels $k \in L_{\text {loc }}^{1}\left(\mathbb{R}_{+}\right)$which is characterized in terms of their Laplace transforms $\mathcal{K}(\lambda)$ as $\lambda \rightarrow 0$, see assumption $(\mathrm{H})$ below.

\subsection{Definitions and Main Assumptions}

Let $S=\{S(t), t \geq 0\}$ be a subordinator without drift starting at zero, that is, an increasing Lévy process starting at zero, see Bertoin [8] for more details. The Laplace transform of $S(t), t \geq 0$ is expressed in terms of a Bernstein function $\Phi:[0, \infty) \longrightarrow[0, \infty)$ (also known as Laplace exponent) by

$$
\mathbb{E}\left(e^{-\lambda S(t)}\right)=e^{-t \Phi(\lambda)}, \quad \lambda \geq 0 .
$$

The function $\Phi$ admits the Lévy-Khintchine representation

$$
\Phi(\lambda)=\int_{(0, \infty)}\left(1-e^{-\lambda \tau}\right) \mathrm{d} \sigma(\tau)
$$

where the measure $\sigma$ (called Lévy measure) has support in $[0, \infty)$ and fulfills

$$
\int_{(0, \infty)}(1 \wedge \tau) \mathrm{d} \sigma(\tau)<\infty
$$

In what follows we assume that the Lévy measure $\sigma$ satisfy

$$
\sigma((0, \infty))=\infty
$$

Using the Lévy measure $\sigma$ we define the kernel $k$ as follows

$$
k:(0, \infty) \longrightarrow(0, \infty), t \mapsto k(t):=\sigma((t, \infty)) .
$$

Its Laplace transform is denoted by $\mathcal{K}$, that is, for any $\lambda \geq 0$ one has

$$
\mathcal{K}(\lambda):=\int_{0}^{\infty} e^{-\lambda t} k(t) \mathrm{d} t
$$

The relation between the function $\mathcal{K}$ and the Laplace exponent $\Phi$ is given by

$$
\Phi(\lambda)=\lambda \mathcal{K}(\lambda), \quad \forall \lambda \geq 0 .
$$

We make the following assumption on the Laplace exponent $\Phi(\lambda)$ of the subordinator $S$.

(H) $\Phi$ is a complete Bernstein function (more precisely, the Lévy measure $\sigma$ has a completely monotone density $\rho(t)$ with respect to the Lebesgue measure, that is, $(-1)^{n} \rho^{(n)}(t) \geq 0$ for all $\left.t>0, n=0,1,2, \ldots\right)$ and the functions $\mathcal{K}, \Phi$ satisfy

$$
\begin{aligned}
& \mathcal{K}(\lambda) \rightarrow \infty, \text { as } \lambda \rightarrow 0 ; \quad \mathcal{K}(\lambda) \rightarrow 0, \text { as } \lambda \rightarrow \infty ; \\
& \Phi(\lambda) \rightarrow 0, \text { as } \lambda \rightarrow 0 ; \quad \Phi(\lambda) \rightarrow \infty, \text { as } \lambda \rightarrow \infty .
\end{aligned}
$$

Example 1. A classical example of a subordinator $S$ is the so-called $\alpha$-stable process with index $\alpha \in(0,1)$. Specifically, a subordinator is $\alpha$-stable if its Laplace exponent is

$$
\Phi(\lambda)=\lambda^{\alpha}=\int_{0}^{\infty}\left(1-e^{-\lambda \tau}\right) \frac{\alpha \tau^{-1-\alpha}}{\Gamma(1-\alpha)} \mathrm{d} \tau .
$$


In this case it follows that the Lévy measure is $\mathrm{d} \sigma_{\alpha}(\tau)=\frac{\alpha}{\Gamma(1-\alpha)} \tau^{-(1+\alpha)} \mathrm{d} \tau$. The corresponding kernel $k_{\alpha}$ has the form $k_{\alpha}(t)=g_{1-\alpha}(t):=\frac{t^{-\alpha}}{\Gamma(1-\alpha)}, t \geq 0$ and its Laplace transform is $\mathcal{K}_{\alpha}(\lambda)=$ $\lambda^{\alpha-1}, \lambda>0$.

Example 2. Sum of two stable subordinators. Let $0<\alpha<\beta<1$ be given and $S_{\alpha, \beta}(t), t \geq 0$ the driftless subordinator with Laplace exponent given by

$$
\Phi_{\alpha, \beta}(\lambda)=\lambda^{\alpha}+\lambda^{\beta}
$$

It is clear from Example 1 that the corresponding Léry measure $\sigma_{\alpha, \beta}$ is the sum of two Lévy measures, that is,

$$
\mathrm{d} \sigma_{\alpha, \beta}(\tau)=\mathrm{d} \sigma_{\alpha}(\tau)+\mathrm{d} \sigma_{\beta}(\tau)=\frac{\alpha}{\Gamma(1-\alpha)} \tau^{-(1+\alpha)} \mathrm{d} \tau+\frac{\beta}{\Gamma(1-\beta)} \tau^{-(1+\beta)} \mathrm{d} \tau .
$$

Then the associated kernel $k_{\alpha, \beta}$ is

$$
k_{\alpha, \beta}(t):=g_{1-\alpha}(t)+g_{1-\beta}(t)=\frac{t^{-\alpha}}{\Gamma(1-\alpha)}+\frac{t^{-\beta}}{\Gamma(1-\beta)}, t>0
$$

and its Laplace transform is $\mathcal{K}_{\alpha, \beta}(\lambda)=\mathcal{K}_{\alpha}(\lambda)+\mathcal{K}_{\beta}(\lambda)=\lambda^{\alpha-1}+\lambda^{\beta-1}, \lambda>0$.

Let $E$ be the inverse process of the subordinator $S$, that is,

$$
E(t):=\inf \{s>0 \mid S(s)>t\}=\sup \{s \geq 0 \mid S(s) \leq t\} .
$$

For any $t \geq 0$ we denote by $G_{t}(\tau), \tau \geq 0$ the marginal density of $E(t)$ or, equivalently

$$
G_{t}(\tau) \mathrm{d} \tau=\frac{\partial}{\partial \tau} P(E(t) \leq \tau) \mathrm{d} \tau=\frac{\partial}{\partial \tau} P(S(\tau) \geq t) \mathrm{d} \tau=-\frac{\partial}{\partial \tau} P(S(\tau)<t) \mathrm{d} \tau .
$$

The density $G_{t}(\tau)$ is the main object in our considerations below. Therefore, in what follows, we collect the most important properties of $G_{t}(\tau)$ needed in the next sections.

Remark 1. If $S$ is the $\alpha$-stable process, $\alpha \in(0,1)$, then the inverse process $E(t)$, has Laplace transform (cf. Prop. 1(a) in Bingham [5] or Feller [6]) given by

$$
\mathbb{E}\left(e^{-\lambda E(t)}\right)=\int_{0}^{\infty} e^{-\lambda \tau} G_{t}(\tau) \mathrm{d} \tau=\sum_{n=0}^{\infty} \frac{\left(-\lambda t^{\alpha}\right)^{n}}{\Gamma(n \alpha+1)}=E_{\alpha}\left(-\lambda t^{\alpha}\right),
$$

where $E_{\alpha}$ is the Mittag-Leffler function. It follows from the asymptotic behavior of the function $E_{\alpha}$ that $\mathbb{E}\left(e^{-\lambda E(t)}\right) \sim C t^{-\alpha}$ as $t \rightarrow \infty$. It is possible to find explicitly the density $G_{t}(\tau)$ in this case using the completely monotonic property of the Mittag-Leffler function $E_{\alpha}$. It is given in terms of the Wright function $W_{\mu, v}$, namely $G_{t}(\tau)=t^{-\alpha} W_{-\alpha, 1-\alpha}\left(\tau t^{-\alpha}\right)$, see Gorenflo et al. [9] for more details.

For a general subordinator, the following lemma determines the $t$-Laplace transform of $G_{t}(\tau)$, with $k$ and $\mathcal{K}$ given in (5) and (6), respectively. For the proof see Kochubei [7] or Proposition 3.2 in Toaldo [2].

Lemma 1. The t-Laplace transform of the density $G_{t}(\tau)$ is given by

$$
\int_{0}^{\infty} e^{-\lambda t} G_{t}(\tau) \mathrm{d} t=\mathcal{K}(\lambda) e^{-\tau \lambda \mathcal{K}(\lambda)} .
$$


The double $(\tau, t)$-Laplace transform of $G_{t}(\tau)$ is

$$
\int_{0}^{\infty} \int_{0}^{\infty} e^{-p \tau} e^{-\lambda t} G_{t}(\tau) \mathrm{d} t \mathrm{~d} \tau=\frac{\mathcal{K}(\lambda)}{\lambda \mathcal{K}(\lambda)+p} .
$$

Here we would like to make the connection of the above abstract framework with general fractional derivatives. For any $\alpha \in(0,1)$ the Caputo-Dzhrbashyan fractional derivative of order $\alpha$ of a function $u$ is defined by (see e.g., Kilbas et al. [10] and references therein)

$$
\left(\mathbb{D}_{t}^{\alpha} u\right)(t)=\frac{d}{d t} \int_{0}^{t} k_{\alpha}(t-\tau) u(\tau) \mathrm{d} \tau-k_{\alpha}(t) u(0), \quad t>0,
$$

where $k_{\alpha}$ is given in Example 1, that is, $k_{\alpha}(t)=g_{1-\alpha}(t)=\frac{t^{-\alpha}}{\Gamma(1-\alpha)}, t>0$. In general, starting with a subordinator $S$ and the kernel $k \in L_{\text {loc }}^{1}\left(\mathbb{R}_{+}\right)$as given in (5), we may define a differential-convolution operator by

$$
\left(\mathbb{D}_{t}^{(k)} u\right)(t)=\frac{d}{d t} \int_{0}^{t} k(t-\tau) u(\tau) \mathrm{d} \tau-k(t) u(0), t>0 .
$$

The operator $\mathbb{D}_{t}^{(k)}$ is also known as general fractional derivative and its applications to convolution-type differential equations was investigated in Kochubei [7].

Example 3. Distributed order derivative. Consider the kernel $k$ defined by

$$
k(t):=\int_{0}^{1} g_{\alpha}(t) \mathrm{d} \alpha=\int_{0}^{1} \frac{t^{\alpha-1}}{\Gamma(\alpha)} \mathrm{d} \alpha, \quad t>0
$$

Then it is easy to see that

$$
\mathcal{K}(\lambda)=\int_{0}^{\infty} e^{-\lambda t} k(t) \mathrm{d} t=\frac{\lambda-1}{\lambda \log (\lambda)}, \quad \lambda>0 .
$$

The corresponding differential-convolution operator $\mathbb{D}_{t}^{(k)}$ is called distributed order derivative, see Atanackovic et al. [11], Daftardar-Gejji and Bhalekar [12], Hanyga [13], Kochubei [14], Gorenflo and Umarov [15], Meerschaert and Scheffler [16] for more details and applications.

We say that the functions $f$ and $g$ are asymptotically equivalent at infinity, and denote $f(x) \sim g(x)$ as $x \rightarrow \infty$, meaning that

$$
\lim _{x \rightarrow \infty} \frac{f(x)}{g(x)}=1 .
$$

We say that a function $L$ is slowly varying at infinity (see Feller [6], Seneta [17]) if

$$
\lim _{x \rightarrow \infty} \frac{L(\lambda x)}{L(x)}=1, \quad \text { for any } \lambda>0 .
$$

Below $C$ is constant whose value is unimportant and may change from line to line.

In the following we consider three classes of admissible kernels $k \in L_{\text {loc }}^{1}\left(\mathbb{R}_{+}\right)$, characterized in terms of their Laplace transforms $\mathcal{K}(\lambda)$ as $\lambda \rightarrow 0$ (i.e., as local conditions):

$$
\begin{gathered}
\mathcal{K}(\lambda) \sim \lambda^{\alpha-1}, \quad 0<\alpha<1 . \\
\mathcal{K}(\lambda) \sim \lambda^{-1} L\left(\frac{1}{\lambda}\right), \quad L(y):=C \log (y)^{-1}, C>0 . \\
\mathcal{K}(\lambda) \sim \lambda^{-1} L\left(\frac{1}{\lambda}\right), \quad L(y):=C \log (y)^{-1-s}, s>0, C>0 .
\end{gathered}
$$


We would like to emphasize that these three classes of kernels leads to different type of differential-convolution operators. In particular, the Caputo-Djrbashian fractional derivative (17) and distributed order derivatives (18), (19). Moreover, it is simple to check that the class of subordinators from Example 2 falls into the class (17) above.

Remark 2. The asymptotic behavior of the function $f(t)$ as $t \rightarrow \infty$ may be determined, under certain conditions, by studying the behavior of its Laplace transform $\tilde{f}(\lambda)$ as $\lambda \rightarrow 0$, and vice versa. An important situation where such a correspondence holds is described by the Feller-Karamata Tauberian (FKT) theorem.

We state below a version of the FKT theorem which suffices for our purposes, see the monographs Bingham et al. [18] (Section 1.7) and Feller [6] (XIII, Section 1.5) for a more general version and proofs.

Theorem 1. Feller-Karamata Tauberian. Let $U:[0, \infty) \longrightarrow \mathbb{R}$ be a monotone non-decreasing right-continuous function such that

$$
w(\lambda):=\int_{0}^{\infty} e^{-\lambda t} \mathrm{~d} U(t)<\infty, \quad \forall \lambda>0 .
$$

If $L$ is a slowly varying function and $C, \rho \geq 0$, then the following are equivalent

$$
\begin{aligned}
& U(t) \sim \frac{C}{\Gamma(\rho+1)} t^{\rho} L(t) \quad \text { as } t \rightarrow \infty, \\
& w(\lambda) \sim C \lambda^{-\rho} L\left(\frac{1}{\lambda}\right) \quad \text { as } \lambda \rightarrow 0^{+} .
\end{aligned}
$$

When $C=0$, (20) is to be interpreted as $U(t)=o\left(t^{\rho} L(t)\right)$; similarly for (21).

\subsection{Statement of the Main Results}

In Section 3 and 4 we will focus our attention on deriving the asymptotic behavior of the subordination $u^{E}(t, x)$ given in (1) for the inverse stable subordinator as well as for the classes (17)-(19) given above. On one hand, the results concerning the inverse stable subordinator as a random time are well understood, due to the fact that the Laplace transform (in $\tau$ ) of the density $G_{t}(\tau)$ is known (cf. Remark 1 ). On the other hand, for a general subordinator much less information about $G_{t}(\tau)$ is known and explicit results for the subordination $u^{E}(t, x)$ are not available. In order to get around this problem, and motivated by the results of Section 3, we study the Cesaro limit of $u^{E}(t, x)$ for the general classes of random times.

With the above considerations we are ready to state our main results.

Theorem 2. Let $u^{E}(t, x)$ be the subordination by the density $G_{t}(\tau)$ associated to the inverse stable subordinator. Denote by $M_{t}\left(u^{E}(\cdot, x)\right):=\frac{1}{t} \int_{0}^{t} u^{E}(s, x) \mathrm{d}$ s the Cesaro mean of $u^{E}(t, x)$.

1. If $u(t, x)=t^{n}, n \geq 0$, then the asymptotic behavior of $u^{E}(t, x)$ coincides with the Cesaro limit and is equal to

$$
C t^{n \alpha} \text { as } t \rightarrow \infty \text {. }
$$

2. If $u(t, x)=e^{-a t}, a>0$, then the asymptotic of $u^{E}(t, x)$ and its Cesaro limit are equal to

$$
C t^{-\alpha} \text { as } t \rightarrow \infty \text {. }
$$

The proof of Theorem 2 is essentially the contents of Section 3 while the next theorem is shown in Section 4.

Theorem 3. Let $u^{E}(t, x)$ be the subordination by the density $G_{t}(\tau)$ associated to the classes (17)-(19) and $M_{t}\left(u^{E}(\cdot, x)\right):=\frac{1}{t} \int_{0}^{t} u^{E}(s, x) \mathrm{d}$ s the Cesaro mean of $u^{E}(t, x)$. 
1. Assume that $u(t, x)=t^{n}, n \geq 0$. Then the asymptotic of the Cesaro mean for the three classes are:

(17). $M_{t}\left(u^{E}(\cdot, x)\right) \sim C t^{\alpha n}$ as $t \rightarrow \infty$,

(18). $M_{t}\left(u^{E}(\cdot, x)\right) \sim C \log (t)^{n}$ as $t \rightarrow \infty$,

(19). $\quad M_{t}\left(u^{E}(\cdot, x)\right) \sim C \log (t)^{(1+s) n}$ as $t \rightarrow \infty$.

2. If $u(t, x)=e^{-a t}, a>0$, then the asymptotic of $M_{t}\left(u^{E}(\cdot, x)\right)$ for the different classes are:

(17). $M_{t}\left(u^{E}(\cdot, x)\right) \sim C t^{-\alpha}$ as $t \rightarrow \infty$,

(18). $M_{t}\left(u^{E}(\cdot, x)\right) \sim C \log (t)^{-1}$ as $t \rightarrow \infty$,

(19). $\quad M_{t}\left(u^{E}(\cdot, x)\right) \sim C \log (t)^{-1-s}$ as $t \rightarrow \infty$.

\section{Inverse Stable Subordinators}

In this section we consider two elementary solutions of dynamical systems, namely $u(t)=u(t, x)=t^{n}, n \geq 0$ and $u(t)=u(t, x)=e^{-a t}, a>0$, and investigate their subordination by the density $G_{t}(\tau)$ of inverse stable subordinator.

Define the function $u^{E}(t)=u^{E}(t, x)$ as the subordination of $u(t)$ (of the above type) by the kernel $G_{t}(\tau)$, that is,

$$
u^{E}(t):=\int_{0}^{\infty} u(\tau) G_{t}(\tau) \mathrm{d} \tau, \quad t \geq 0 .
$$

Our goal is to investigate the asymptotic behavior of $u^{E}(t)$. At first we compute explicitly the function $u^{E}(t)$ by solving the integral (22) and obtain the time asymptotic. Second we derive the Cesaro limit of $u^{E}(t)$, more precisely, the asymptotic behavior of the Cesaro mean of $u^{E}(t)$ defined by

$$
M_{t}\left(u^{E}(\cdot)\right):=\frac{1}{t} \int_{0}^{t} u^{E}(s) \mathrm{d} s .
$$

It turns out that both asymptotic behaviors for the two functions $u(t)$ given above coincide. Therefore, for the random time change associated to the inverse stable subordinator $E(t)$, $t \geq 0$, the asymptotic behavior of $u^{E}(t)$ is the same as the Cesaro limit. On the other hand, using the Cesaro limit we may investigate a broad class of subordinators. In Section 4 we investigate the Cesaro limit for the classes (17)-(19) while in this section concentrate in the spacial case of inverse stable subordinators.

\subsection{Subordination of Monomials}

Let us consider at first the subordination of the function $u(t)=t^{n}, n \geq 0$. Hence, $u^{E}(t)$ is given by

$$
u^{E}(t)=\int_{0}^{\infty} \tau^{n} G_{t}(\tau) \mathrm{d} \tau .
$$

It follows from (11) that $u^{E}(t)$ is explicitly evaluated as

$$
u^{E}(t)=\left.(-1)^{n} \frac{\mathrm{d}^{n}}{\mathrm{~d} \lambda^{n}} E_{\alpha}\left(-\lambda t^{\alpha}\right)\right|_{\lambda=0}=\frac{n !}{\Gamma(\alpha n+1)} t^{\alpha n} .
$$

The last equality follows easily from the power series of the Mittag-Leffler function

$$
E_{\alpha}(z)=\sum_{n=1}^{\infty} \frac{z^{n}}{\Gamma(\alpha n+1)} .
$$

In addition, the asymptotic of the Mittag-Leffler function $E_{\alpha}$ that gives

$$
u^{E}(t) \sim C t^{n \alpha} \text { as } t \rightarrow \infty .
$$


Now we turn to compute the asymptotic behavior of the Cesaro mean of $u^{E}(t)$ with the help of the FKT theorem. To this end we define the monotone function

$$
v(t):=\int_{0}^{t} u^{E}(s) \mathrm{d} s
$$

The Laplace-Stieltjes transform $w(\lambda)$ of $v(t)$ is given by

$$
w(\lambda):=\int_{0}^{\infty} e^{-\lambda t} \mathrm{~d} v(t)=\int_{0}^{\infty} e^{-\lambda t} u^{E}(t) \mathrm{d} t=\int_{0}^{\infty} e^{-\lambda t} \int_{0}^{\infty} \tau^{n} G_{t}(\tau) \mathrm{d} \tau \mathrm{d} t .
$$

Using Fubini's theorem and Equation (12) we obtain

$$
w(\lambda)=\int_{0}^{\infty} \tau^{n} \int_{0}^{\infty} e^{-\lambda t} G_{t}(\tau) \mathrm{d} t \mathrm{~d} \tau=\mathcal{K}(\lambda) \int_{0}^{\infty} \tau^{n} e^{-\tau \lambda \mathcal{K}(\lambda)} \mathrm{d} \tau .
$$

The r.h.s. integral can be evaluated as

$$
\int_{0}^{\infty} \tau^{n} e^{-\tau \lambda \mathcal{K}(\lambda)} \mathrm{d} \tau=(\lambda \mathcal{K}(\lambda))^{-(1+n)} n !
$$

which yields

$$
w(\lambda)=n ! \lambda^{-(1+n)} \mathcal{K}(\lambda)^{-n}
$$

On the other hand, for the stable subordinator we have $\mathcal{K}(\lambda)=\lambda^{\alpha-1}$, cf. Example 1. Thus, we obtain

$$
w(\lambda)=n ! \lambda^{-(1+\alpha n)}=\lambda^{-\rho} L\left(\frac{1}{\lambda}\right)
$$

where $\rho=1+\alpha n$ and $L(x)=n$ ! is a trivial slowly varying function. Then Theorem 1 yields

$$
v(t) \sim C t^{1+n \alpha} \quad \text { as } t \rightarrow \infty
$$

and this implies the following asymptotic behavior for the Cesaro mean of $u^{E}(t)$

$$
M_{t}\left(u^{E}(\cdot)\right)=\frac{1}{t} \int_{0}^{t} u^{E}(s) \mathrm{d} s \sim C t^{\alpha n} \quad \text { as } \quad t \rightarrow \infty .
$$

Remark 3. In conclusion, we find that the asymptotic behavior of the subordination $u^{E}(t)$ of any monomial by the density $G_{t}(\tau)$ (of the inverse stable subordinator) as well as its Cesaro limit coincides. Note also the slower decay of the subordination $u^{E}(t)$ compared to $u(t)$ due to $0<\alpha<1$.

\subsection{Subordination of Decaying Exponentials}

Now we consider the solution $u(t)=e^{-a t}, a>0$ and proceed to study the asymptotic behavior of its subordination $u^{E}(t)$ by the kernel $G_{t}(\tau)$. Again a direct computation is possible in that case as well as the Cesaro mean.

Hence, the subordination $u^{E}(t)$ is given by

$$
u^{E}(t)=\int_{0}^{\infty} u(\tau) G_{t}(\tau) \mathrm{d} \tau=\int_{0}^{\infty} e^{-a \tau} G_{t}(\tau) \mathrm{d} \tau
$$

It follows from Equation (11) that

$$
u^{E}(t)=E_{\alpha}\left(-a t^{\alpha}\right) \sim C t^{-\alpha} \quad \text { as } \quad t \rightarrow \infty .
$$

On the other hand, to derive the asymptotic behavior for the Cesaro mean of $u^{E}(t)$ (with the help of Theorem 1) we define the monotone function

$$
v(t):=\int_{0}^{t} u^{E}(s) \mathrm{d} s
$$


The Laplace-Stieltjes transform $w(\lambda)$ of $v(t)$ is equal to

$$
w(\lambda):=\int_{0}^{\infty} e^{-\lambda t} \mathrm{~d} v(t)=\int_{0}^{\infty} e^{-\lambda t} u^{E}(t) \mathrm{d} t=\int_{0}^{\infty} e^{-\lambda t} \int_{0}^{\infty} e^{-a \tau} G_{t}(\tau) \mathrm{d} \tau \mathrm{d} t
$$

and using Fubini's theorem and Equation (11) we obtain

$$
w(\lambda)=\mathcal{K}(\lambda) \int_{0}^{\infty} e^{-\tau(a+\lambda \mathcal{K}(\lambda))} \mathrm{d} \tau=\frac{\mathcal{K}(\lambda)}{a+\lambda \mathcal{K}(\lambda)} .
$$

As $\mathcal{K}(\lambda)=\lambda^{\alpha-1}$ for the class (17) we may write $\tilde{v}(\lambda)$ as

$$
w(\lambda)=\lambda^{-(1-\alpha)} \frac{1}{a+\lambda^{\alpha}}=\lambda^{-\rho} L\left(\frac{1}{\lambda}\right), \quad \rho=1-\alpha, \quad L(t):=\frac{1}{a+t^{-\alpha}} .
$$

It is simple to verify that $L$ is a slowly varying function so that we may use the FKT theorem to obtain

$$
v(t) \sim C t^{1-\alpha} \frac{1}{a+t^{-\alpha}} \quad \text { as } t \rightarrow \infty .
$$

Dividing both sides by $t$ leads to the asymptotic behavior of the Cesaro mean of $u^{E}(t)$, that is,

$$
M_{t}\left(u^{E}(\cdot)\right)=\frac{1}{t} \int_{0}^{t} u^{E}(s, x) \mathrm{d} s \sim C \frac{t^{-\alpha}}{a+t^{-\alpha}} \sim C t^{-\alpha} \quad \text { as } t \rightarrow \infty .
$$

Remark 4. We conclude that the asymptotic behavior $u^{E}(t)$ given in (30) coincides with the Cesaro limit of $u^{E}(t, x)$. In addition, we notice that the starting function $u(t)=e^{-a t}$ has an exponential decay and its subordination has a slower decay, namely polynomial decay.

\section{Cesaro Limit for General Classes of Subordinators}

In this section we study the asymptotic behavior of the subordination by the density $G_{t}(\tau)$ associated to the classes (17)-(19). Note that Examples 1 and 2 belong to the class (17). As pointed out in Section 3 here we only study the Cesaro limit of the subordination function $u^{E}(t)$.

As in Section 3, $u^{E}(t)$ is defined by

$$
u^{E}(t):=\int_{0}^{\infty} \tau^{n} G_{t}(\tau) \mathrm{d} \tau
$$

or

$$
u^{E}(t):=\int_{0}^{\infty} e^{-a \tau} G_{t}(\tau) \mathrm{d} \tau
$$

while $v(t)$ is defined by

$$
v(t):=\int_{0}^{t} u^{E}(s) \mathrm{d} s
$$

The density $G_{t}(\tau)$ in (34) and (35) is associated to each class (17)-(19) described above. We study the Cesaro limit of $u^{E}(t)$ for each class separately.

\subsection{Subordination by the Class (17)}

At first we study the asymptotic behavior of $u^{E}(t)$ given by (34). To this end we use equality (27) to obtain the Laplace-Stieltjes transform $w(\lambda)$ of the function $v(t)$ as

$$
w(\lambda):=\int_{0}^{\infty} e^{-\lambda t} \mathrm{~d} v(t)=\lambda^{-(1+n)}(\mathcal{K}(\lambda))^{-n} n !
$$

It follows from the behavior of $\mathcal{K}(\lambda)$ at $\lambda=0$ of the class (17) that

$$
w(\lambda) \sim \lambda^{-(1+\alpha n)} n !=\lambda^{-\rho} L\left(\frac{1}{\lambda}\right)
$$


where $\rho=1+\alpha n$ and $L(x)=n$ ! is a slowly varying function. It follows from the FKT theorem that

$$
v(t) \sim C t^{\rho} L(t)=C t^{1+\alpha n} \quad \text { as } \quad t \rightarrow \infty .
$$

This implies the Cesaro limit of $u^{E}(t)$ as

$$
M_{t}\left(u^{E}(\cdot)\right) \sim C t^{\alpha n} \quad \text { as } t \rightarrow \infty .
$$

Note that this asymptotic is similar to the analogous for the inverse stable subordinator, cf. (28).

Let us now study the Cesaro limit of the function $u^{E}(t)$ given in (35). Using the equality (32) the Laplace-Stieltjes transform $v(t)$ has the form

$$
\tilde{v}(\lambda)=\frac{\mathcal{K}(\lambda)}{a+\lambda \mathcal{K}(\lambda)}
$$

Replacing the local behavior of $\mathcal{K}(\lambda)$ at $\lambda=0$ for the class (17) gives

$$
\tilde{v}(\lambda) \sim \frac{\lambda^{\alpha-1}}{a+\lambda^{\alpha}}=\lambda^{-\rho} L\left(\frac{1}{\lambda}\right)
$$

where $\rho=1-\alpha$ and $L(x)=\frac{1}{1+a x^{-\alpha}}$. An applications of the FKT theorem yields the asymptotic for $v(t)$, namely $v(t) \sim C t^{\rho} L(t)$ as $t \rightarrow \infty$. Finally dividing both sides by $t$ gives the Cesaro limit of $u^{E}(t)$, that is,

$$
M_{t}\left(u^{E}(\cdot)\right) \sim C \frac{t^{-\alpha}}{1+a t^{-\alpha}} \sim C t^{-\alpha} \quad \text { as } \quad t \rightarrow \infty .
$$

Again, we obtain the same asymptotic as for the inverse stable subordinator, see (33). In any case, since $0<\alpha<1$, the time decaying is slower than the initial function $u(t)$.

\subsection{Subordination by the Class (18)}

Assume that $u^{E}(t)$ is the subordination given in (34). The Laplace-Stieltjes transform $w(\lambda)$ of $v(t)$ (cf. equality (27)) has the form

$$
w(\lambda):=\int_{0}^{\infty} e^{-\lambda t} \mathrm{~d} v(t)=\lambda^{-(1+n)}(\mathcal{K}(\lambda))^{-n} n !
$$

Using the behavior of $\mathcal{K}(\lambda)$ near $\lambda=0$ for the class (18) we obtain

$$
w(\lambda) \sim \lambda^{-1} L\left(\frac{1}{\lambda}\right)
$$

where $L(x)=C \log (x)^{n}, C>0$, is a slowly varying function. Then it follows from the FKT theorem that

$$
v(t) \sim C t \log (t)^{n}
$$

and as a result the asymptotic behavior for the Cesaro mean of $u^{E}(t)$ follows

$$
M_{t}\left(u^{E}(\cdot)\right) \sim C \log (t)^{n} \quad \text { as } \quad t \rightarrow \infty .
$$

A similar analysis may be applied to study the asymptotic behavior for the subordination $u^{E}(t)$ given in (35). The Laplace-Stieltjes transform $w(\lambda)$ of the monotone function $v(t)$ may be evaluated using equality (32) to find the following expression

$$
w(\lambda)=\frac{\mathcal{K}(\lambda)}{a+\lambda \mathcal{K}(\lambda)}
$$


Using the local behavior of $\mathcal{K}(\lambda)$ near $\lambda=0$ from class (18) yields

$$
w(\lambda) \sim \lambda^{-1} L\left(\frac{1}{\lambda}\right)
$$

where $L(x)=C \frac{\log (x)^{-1}}{a+C \log (x)^{-1}}$ which is a slowly varying function. Using the FKT theorem we obtain the longtime behavior for the Cesaro mean of $u^{E}(t)$ as

$$
M_{t}\left(u^{E}(\cdot)\right) \sim C \frac{\log (t)^{-1}}{a+C \log (t)^{-1}} \sim C \log (t)^{-1} \text { as } t \rightarrow \infty .
$$

\subsection{Subordination by the Class (19)}

At first we study the subordination $u^{E}(t)$ given in (34) for the class (19). The LaplaceStieltjes transform $w(\lambda)$ of the corresponding $v(t)$ is computed using equality (27) and we obtain

$$
w(\lambda):=\int_{0}^{\infty} e^{-\lambda t} \mathrm{~d} v(t)=\lambda^{-(1+n)}(\mathcal{K}(\lambda))^{-n} n !
$$

Using the behavior of $\mathcal{K}(\lambda)$ near $\lambda=0$ for the class (19) yields

$$
w(\lambda) \sim \lambda^{-1} L\left(\frac{1}{\lambda}\right)
$$

where $L(x)=C \log (x)^{(1+s) n}, C>0$, is a slowly varying function. Then it follows from Theorem 1 that

$$
v(t) \sim C t \log (t)^{(1+s) n}
$$

and dividing both sides by $t$ gives the asymptotic behavior for the Cesaro mean of $u^{E}(t)$, namely

$$
M_{t}\left(u^{E}(\cdot)\right) \sim C \log (t)^{(1+s) n} \quad \text { as } t \rightarrow \infty .
$$

Let $u^{E}(t)$ be the subordination by $u(t)=e^{-a t}, a>0$, that is, equality (35) with $G_{t}(\tau)$ from the class (19). It follows from equality (32) that the Laplace-Stieltjes transform $w(\lambda)$ of $v(t)$ has the form

$$
w(\lambda)=\frac{\mathcal{K}(\lambda)}{a+\lambda \mathcal{K}(\lambda)} .
$$

Using the local behavior of $\mathcal{K}(\lambda)$ near $\lambda=0$ from class (19) yields

$$
w(\lambda) \sim \lambda^{-1} L\left(\frac{1}{\lambda}\right), \quad L(x)=C \frac{\log (x)^{-1-s}}{a+C \log (x)^{-1-s}},
$$

where $C, s>0$. As the function $L$ is slowly varying at infinity, then by the FKT theorem we obtain the asymptotic behavior for the Cesaro mean of $u^{E}(t)$ as

$$
M_{t}\left(u^{E}(\cdot)\right) \sim C \frac{\log (t)^{-1-s}}{a+C \log (t)^{-1-s}} \sim C \log (t)^{-1-s} \quad \text { as } \quad t \rightarrow \infty .
$$

Author Contributions: Writing—original draft preparation, Y.K. and J.d.S. All authors have read and agreed to the published version of the manuscript.

Funding: This research was funded by Fundação para a Ciência e a Tecnologia, Portugal grant number UIDB/MAT/04674/2020 and Ministry of Education and Science of Ukraine grant number 0119 U002583.

Institutional Review Board Statement: Not applicable.

Informed Consent Statement: Not applicable. 
Conflicts of Interest: The authors declare no conflict of interest.

\section{References}

1. Kondratiev, Y.; da Silva, J. Random Time Dynamical Systems I: General Structures. arXiv 2020, arXiv:2012.15201v1.

2. Toaldo, B. Convolution-type derivatives, hitting-times of subordinators and time-changed $C_{0}$-semigroups. Potential Anal. 2015, 42, 115-140. [CrossRef]

3. Meerschaert, M.M.; Sikorskii, A. Stochastic Models for Fractional Calculus; Walter de Gruyter \& Co.: Berlin, Germany, 2012; Volume 43.

4. Mura, A.; Taqqu, M.S.; Mainardi, F. Non-Markovian diffusion equations and processes: analysis and simulations. Phys. A 2008, 387, 5033-5064. [CrossRef]

5. Bingham, N.H. Limit theorems for occupation times of Markov processes. Z. Wahrscheinlichkeitstheorie Verwandte Geb. 1971, 17, 1-22. [CrossRef]

6. Feller, W. An Introduction to Probability Theory and Its Applications, 2nd ed.; John Wiley \& Sons Inc.: New York, NY, USA, 1971; Volume II.

7. Kochubei, A.N. General Fractional Calculus, Evolution Equations, and Renewal Processes. Integral Equ. Oper. Theory 2011, 71,583-600. [CrossRef]

8. Bertoin, J. Lévy Processes; Cambridge Tracts in Mathematics; Cambridge University Press: Cambridge, UK, 1996; Volume 121, p. $x+265$.

9. Gorenflo, R.; Luchko, Y.; Mainardi, F. Analytical properties and applications of the Wright function. Fract. Calc. Appl. Anal. 1999, 2, 383-414.

10. Kilbas, A.A.; Srivastava, H.M.; Trujillo, J.J. Theory and Applications of Fractional Differential Equations; North-Holland Mathematics Studies; Elsevier Science B.V.: Amsterdam, The Netherlands, 2006; Volume 204.

11. Atanackovic, T.M.; Pilipovic, S.; Zorica, D. Time distributed-order diffusion-wave equation. I., II. Proceedings of the Royal Society of London A: Mathematical, Physical and Engineering Sciences. R. Soc. 2009, 465, 869-1891.

12. Daftardar-Gejji, V.; Bhalekar, S. Boundary value problems for multi-term fractional differential equations. J. Math. Anal. Appl. 2008, 345, 754-765. [CrossRef]

13. Hanyga, A. Anomalous diffusion without scale invariance. J. Phys. A Mat. Theor. 2007, 40, 5551. [CrossRef]

14. Kochubei, A.N. Distributed order calculus and equations of ultraslow diffusion. J. Math. Anal. Appl. 2008, 340, 252-281. [CrossRef]

15. Gorenflo, R.; Umarov, S. Cauchy and nonlocal multi-point problems for distributed order pseudo-differential equations, Part one. Z. Anal. Anwend. 2005, 24, 449-466. [CrossRef]

16. Meerschaert, M.M.; Scheffler, H.P. Stochastic model for ultraslow diffusion. Stoch. Process. Appl. 2006, 116, 1215-1235. [CrossRef]

17. Seneta, E. Regularly Varying Functions; Lect. Notes Math.; Springer: Berlin/Heidelberg, Germany, 1976; Volume 508.

18. Bingham, N.H.; Goldie, C.M.; Teugels, J.L. Regular Variation; Encyclopedia of Mathematics and its Applications; Cambridge University Press: Cambridge, UK, 1987; Volume 27. 